\title{
BMJ Open A prospective, open-label, interventional study protocol to evaluate treatment efficacy of nivolumab based on serum-soluble PD-L1 concentration for patients with metastatic and unresectable renal cell carcinoma
}

\author{
Yukari Bando, ${ }^{1}$ Nobuyuki Hinata (D) , ${ }^{1}$ Takashi Omori, ${ }^{2}$ Masato Fujisawa ${ }^{1}$
}

To cite: Bando Y, Hinata N, Omori T, et al. A prospective, open-label, interventional study protocol to evaluate treatment efficacy of nivolumab based on serum-soluble PD-L1 concentration for patients with metastatic and unresectable renal cell carcinoma. BMJ Open 2019;9:e030522. doi:10.1136/ bmjopen-2019-030522

- Prepublication history for this paper is available online To view these files, please visit the journal online (http://dx.doi. org/10.1136/bmjopen-2019030522).

Received 23 March 2019 Revised 01 November 2019 Accepted 07 November 2019

\section{Check for updates}

(c) Author(s) (or their employer(s)) 2019. Re-use permitted under CC BY-NC. No commercial re-use. See rights and permissions. Published by BMJ.

${ }^{1}$ Department of Urology, Kobe University Graduate School of Medicine School of Medicine, Kobe, Hyogo, Japan

${ }^{2}$ Center Administration Division, Center for Clinical Research, Kobe University Hospital, Kobe, Japan

Correspondence to Dr Nobuyuki Hinata; hinata@med.kobe-u.ac.jp

\section{ABSTRACT}

Introduction Nivolumab has been proven to prolong overall survival as a second-line therapy for patients with advanced renal cell carcinoma (RCC) in a phase III clinical trial. However, versatile biomarkers have not been established to predict the efficacy of nivolumab against target disease.

Methods and analysis After registration, screening test and serum-soluble programmed cell death 1-ligand 1 (SPD-L1) measurement will be performed by using the ELISA; patients will be grouped into high SPD-L1 or low SPD-L1 groups. Nivolumab (240 mg every 2 weeks by intravenous drip infusion) will be administered to each participant. For this prospective study, statistical power calculation indicated that 48 participants with metastatic or unresectable RCC are needed to assess the efficacy of this method. The participants must be at the age of at least 20 years at the time of informed consent and require second-line therapy after failure of first-line therapy or discontinuation due to adverse effects. All data will be collected in our institution. The primary endpoint is progression-free survival, and secondary endpoints are overall survival and objective response rate. In this protocol, we will examine SPD-L1 as a promising predictive marker.

Ethics and dissemination This protocol was approved by the Kobe University Clinical Research Ethical Committee (C180067). Findings of this study will be widely disseminated through conference presentations, reports, factsheets and academic publications; further generalisation will also be discussed.

Trial registration number UMIN000027873.

\section{INTRODUCTION}

Renal cell carcinoma (RCC), a tumour arising in the kidney parenchyma, is a malignant renal disease of high frequency. Nivolumab has been proven to prolong overall survival as a second-line therapy for patients with advanced RCC in a phase III clinical trial. In major guidelines, nivolumab is strongly

\section{Strengths and limitation of this study}

- The association between the efficacy of nivolum$\mathrm{ab}$ and soluble programmed cell death 1-ligand 1 (sPD-L1) is explored for the first time in patients of advanced renal cell carcinoma.

- SPD-L1 is measured easily and with low invasiveness and may provide significant benefits to patients of advanced renal cell carcinoma if the association with the efficacy of nivolumab is revealed.

- Our study population is of limited sample size though the sample size is strictly designed.

recommended as second-line therapy for advanced RCC. ${ }^{23}$ However, biomarkers have not been established to predict the efficacy of nivolumab against target disease. ${ }^{4}$

Programmed cell death-1 ligand-1 (PDL1) is an inhibitory co-stimulatory factor that is expressed on antigen-presenting cells, which induces lymphocyte inactivation and apoptosis via the receptor programmed cell death-1 (PD-1). In recent years, for a variety of malignant tumours, a relationship has been noted between hyperactivation of PD-L1 expression on antigen-presenting cells or tumour cells and the degree of tumour malignancy ${ }^{5-7}$ However, most of these reports evaluated the expression of PD-L1 or PD-1 by immunohistochemistry or flow cytometry. These approaches are lacking in convenience and speed, exhibit wide variation in their results, and do not involve standard evaluation methods. Therefore, a novel examination method is needed to evaluate the expression of PD-L1.

The expression of soluble PD-L1 reportedly shows a positive correlation with the expression of serum PD-L1 on antigen-presenting 
cells. $^{8}$ In RCC, the expression of PD-L1 in tumour tissue comprises a prognostic predictor. ${ }^{9}$ Furthermore, the concentration of serum-soluble PD-L1 is reportedly higher in more aggressive renal cell carcinoma. ${ }^{10}$ Therefore, we aim to examine the therapeutic effect and prognosis of patients with high concentrations of serum-soluble PD-L1, compared with the therapeutic effect and prognosis of patients with low concentrations of serum-soluble PD-L1. The hypothesis of this study is that patients with high blood levels of serum-soluble PD-L1 will experience a greater therapeutic effect during nivolumab treatment.

\section{METHODS AND ANALYSIS \\ Study objective}

The objective of this prospective study is to investigate the effectiveness of nivolumab in patients with metastatic and/or unresectable RCC with high blood levels of serum-soluble PD-L1, using patients with low blood levels of serum-soluble PD-L1 as a control group.

\section{Study design and setting}

This study is a prospective single-centre, single-arm, openlabel invasive intervention study, with a 2-year enrolment period and 5-year follow-up period, which is performed at the Kobe University Hospital. Patient recruitment commenced on 1 November 2017 and was completed on 30 April 2019. The data are expected to be available no earlier than 30 April 2024.

\section{Participants}

The following eligibility criteria will be used in this study:

1. Advanced RCG.

2. Metastatic and/or unresectable RCC (radiologically or pathologically confirmed).

3. Age of at least 20 years at the time of informed consent.
4. Written consent for participation is provided by the patient.

5. Requirement for second-line therapy after failure of first-line therapy or discontinuation due to adverse effects.

Exclusion criteria

1. Presence of active double cancer.

2. Ongoing pregnancy or lactation.

3. Presence of mental illness or psychiatric symptoms that may interfere with participation in the study.

4. Drug hypersensitivity to components of nivolumab.

5. Ineligibility to participate, as determined by clinical physicians in the study, for other reasons.

6. Presence of severe autoimmune disease and use of chronic corticosteroid medication.

\section{Ethical approval and consent to study participation}

Written informed consent to participate in this study will be obtained from all patients by clinical physicians. The clinical physicians will provide potential participants with opportunities to ask questions and sufficient time to consider whether to provide consent; they will also confirm that potential participants have sufficient understanding of this study, before obtaining voluntary consent from the potential participants. The flowchart of this study is summarised in figure 1.

\section{Outcome measures}

The primary endpoint in this study will be progressionfree survival, defined as the period from the first administration of nivolumab until confirmation of exacerbation. Because it reflects clinically important overall survival time and can be to make an early determination of prognosis, this outcome measure was chosen as the primary endpoint. Secondary endpoints include overall survival, which is the period from the first administration of
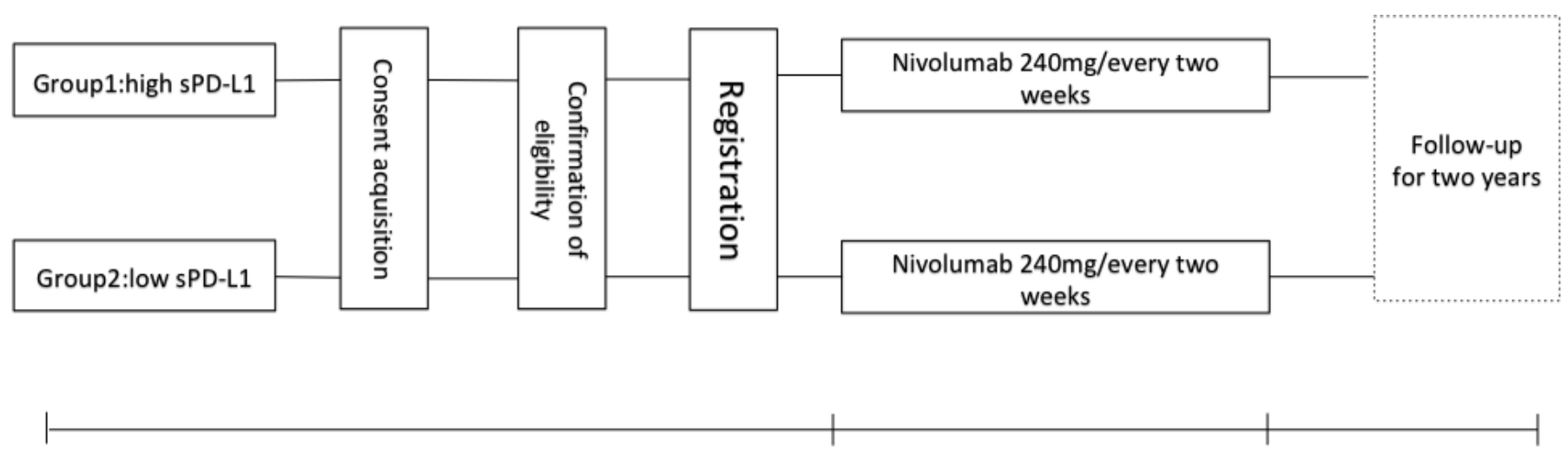

-Wash-out and observation

Treatment

Observation $\longrightarrow$

$\longleftarrow$ blood test $(4,12,24,48$ weeks $) \longrightarrow$

Figure 1 Flowchart of this study protocol. 
Table 1 Follow-up schedule for data collection

\begin{tabular}{|c|c|c|c|c|c|c|c|}
\hline Screening & Period & $\begin{array}{l}\text { Oweek } \\
\text { (day 0) }\end{array}$ & $\begin{array}{l}4 \text { weeks } \\
\text { (day } 28 \text { ) }\end{array}$ & $\begin{array}{l}12 \text { weeks } \\
\text { (day 84) }\end{array}$ & Discontinuation & $\begin{array}{l}24 \text { weeks } \\
\text { (day 168) }\end{array}$ & $\begin{array}{l}48 \text { weeks } \\
\text { (day } 336 \text { ) }\end{array}$ \\
\hline $\begin{array}{l}\text { Range of aberration } \\
\text { (day) }\end{array}$ & -14 & 0 & \pm 3 & \pm 7 & \pm 7 & \pm 7 & \\
\hline Measurement of sPD-L1 & $\bullet$ & & 0 & 0 & 0 & $\bullet$ & 0 \\
\hline $\begin{array}{l}\text { Diameter of target lesion } \\
\text { (secondary endpoint ) }\end{array}$ & 0 & & 0 & 0 & 0 & ○ & 0 \\
\hline General blood test ${ }^{*}$ & ○ & & 0 & 0 & 0 & 0 & 0 \\
\hline Urine check & 0 & & 0 & 0 & 0 & 0 & 0 \\
\hline
\end{tabular}

*General blood test includes WBC, RBC, Hb, Ht, MCV,MCH, MCHC, Plt.

SPD-L1, soluble programmed cell death 1-ligand 1.

nivolumab until confirmation of death, and objective response rate (complete response +partial response, based on the RECIST criteria $^{10}$ ). Overall survival time is clinically important; thus, secondary endpoints that reflect the proximity effect of nivolumab were chosen as secondary endpoints. The occurrence of adverse events during the study period constituted the safety endpoint.

\section{Schedule}

\section{Research outline}

After registration, screening test and serum-soluble PD-L1 measurement by ELISA will be performed; patients will be grouped on the basis of serum-soluble PD-L1 concentrations (hereinafter referred to as high sPD-L1 and low sPD-L1 groups). The cut-off value is set at $0.23 \mathrm{ng} / \mathrm{mL}$, which was the median concentration of soluble PD-L1 measured in patients with RCC in a prior study. ${ }^{11}$

Nivolumab will be administered to each participant within 14 days thereafter. We will collect blood clinical data before nivolumab treatment, then after 4, 12, 24 and 48 weeks of treatment. The status of each patient will be followed for 2 years with respect to response rate, disease progression and death. The schedule for observations, tests and assessments is shown in table 1; all will be performed by clinical physicians as indicated.

\section{Dosage regimen, dose and administration period}

\section{Prohibited medicine for concomitant use}

Live vaccines, attenuated live vaccines and inactivated vaccines will be prohibited because unexpected symptoms may occur due to the presence of an excessive immune response accompanying T-cell activation after administration of nivolumab.

\section{Case registration and allocation}

1. A clinical physician involved in the study will perform a screening test within 7 days after acquiring informed consent from a potential participant, in order to determine the participant's eligibility.

2. If eligibility is confirmed, the participant will be registered and the clinical physician will promptly use the case-registration application form to record the participant's identification code, sex, age, date of consent and scheduled administration start date; this form will be submitted to the secretariat.

3. The research secretariat will record drug assignment and provide a confirmation letter to the clinical physician.

4. The clinical physician will administer medicine to the participant within 14 days after the screening test.

\section{Aftercare to participants}

In accordance with the Declaration of Helsinki and Clinical Research Law, the researchers will ensure that the participants receive the best prevention, diagnosis and treatment available, based on the results of the study, even after the study has ended.

\section{Sample management}

The researchers will preserve samples for 5 years from the date reported on the final public announcement of the results of this study. Samples will then be discarded in a manner that avoids identification of the participants. The sample and information obtained will not be used for purposes other than this study. Provision of samples and information to other institutions will be prohibited.

\section{Data management}

The clinical physicians will confirm that each potential participant meets the inclusion criteria and will complete corresponding registration forms. Each participant will be assigned an identification code when all the registration forms are submitted to the research executive office. The research director will prepare a coded list to facilitate the identification of participants during and after the research. As necessary, the clinical physicians will confirm the validity of this document. The code list confidentiality will be maintained, and the research director will retain the list in the research file. 


\section{Statistical analyses}

\section{Sample size of primary endpoints}

A sample size calculation was performed on the basis of estimated progression-free survival time of nivolumabtreated patients with advanced RCC in a Phase III clinical study (4) (mean, 4.6 months; 95\% CI 3.7 to 5.4 months). We assumed that the median progression-free survivals of the high sPD-L1 group and low sPD-L1 groups would be 8 months and 3 months, respectively. To detect this difference with $80 \%$ power using a two-tailed test and a $5 \%$ significance level, 24 participants per group are needed (total 48 participants).

\section{Planned statistical analysis of baseline characteristics}

The distribution of participant baseline characteristics in each group will be described by summary statistics, such as median and frequency. Differences between groups of patients will be tested with the Wilcoxon rank-sum test for quantitative variables; differences between groups of patients will be tested with chi-squared test for categorical variable variables. Differences in adverse events between two groups will be tested with Fisher's exact test.

\section{Planned statistical analysis of primary endpoints}

Progression-free survivals will be estimated by the Kaplan-Meier method, and the resulting distribution will be compared using a log-rank test. Differences will be regarded as statistically significant at $\mathrm{p}<0.05$.

\section{Planned statistical analysis of secondary endpoints}

Secondary endpoints will be analysed to supplement assessment of the primary endpoint. The response rate will analysed using the chi-squared test. The percentage difference and its $95 \%$ CI will be estimated. Overall survival will be estimated by the Kaplan-Meier method, and the resulting distribution will be compared using a log-rank test. Differences will be regarded as statistically significant at $\mathrm{p}<0.05$.

\section{Analysis of safety}

Adverse events will be summarised by using frequency and percentage, and a summary table will be prepared. The analysis of safety will be performed for the participants that receive at least one administration of nivolumab.

\section{Quality assurance}

Monitoring will be performed periodically to ensure that this research is carried out safely and in accordance with the implementation plan, as well as to determine whether data are accurately recorded and stored. This work will be supervised by dedicated staff members.

\section{Patient and public involvement}

Patients and public were not involved in the design of this study. Moreover, they will not be involved in the recruitment to the study, nor the conduct of the study.

\section{DISCUSSION}

Nivolumab has been proven to prolong overall survival as a second-line therapy for patients with advanced
RCG in a Phase III clinical trial. ${ }^{1}$ However, versatile biomarkers have not been established to predict the efficacy of nivolumab against target disease. ${ }^{4}$ In prior studies, sPD-L1 was reported to exhibit a positive correlation with the aggressiveness of RCC. Therefore, we hypothesise that in patients with high serumsoluble PD-L1 concentration, the therapeutic effect of nivolumab will be greater.

This study is a single-arm, exploratory clinical trial, which may be a limitation. With this study design, treatment bias cannot be completely excluded. Moreover, in terms of comparison between groups, it is difficult to ensure that patients have similar backgrounds.

In conclusion, there have been few prospective studies of predictive biomarkers for the effectiveness of nivolumab. Thus, a prospective study is needed to determine the feasibility and efficacy of sPD-L1.

\section{ETHICS AND DISSEMINATION}

Findings of this study will be widely disseminated through conference presentations, reports, factsheets and academic publications; further generalisations will also be discussed.

Acknowledgements We thank Ryan Chastain-Gross, Ph.D., from Edanz Group ( www.edanzediting.com/ac) for editing a draft of this manuscript.

Contributors MF is the principal investigator of this study. NH designed the study concept and supervised the study. YB is the study coordinator and drafted the manuscript. TO is the biostatistician who contributed to statistical analysis of historical control data and calculation of the sample size. All authors read and approved the final manuscript.

Funding This work was supported by Ono Pharmaceutical Co., Ltd. and BristolMyers Squibb, Co.

Competing interests None declared.

Patient consent for publication Not required.

Ethics approval The study protocol version 4.0 (C180067-290020) has been approved by the ethical review board at our institution. This study conforms to the provisions of the revised Declaration of Helsinki, the Ethical Guidelines for Medical and Health Research Involving Human Subjects in Japan, and the Clinical Trial Act in Japan.

Provenance and peer review Not commissioned; externally peer reviewed.

Open access This is an open access article distributed in accordance with the Creative Commons Attribution Non Commercial (CC BY-NC 4.0) license, which permits others to distribute, remix, adapt, build upon this work non-commercially, and license their derivative works on different terms, provided the original work is properly cited, appropriate credit is given, any changes made indicated, and the use is non-commercial. See: http://creativecommons.org/licenses/by-nc/4.0/.

ORCID iD

Nobuyuki Hinata http://orcid.org/0000-0001-7014-6812

\section{REFERENCES}

1 Motzer RJ, Escudier B, McDermott DF, et al. Nivolumab versus everolimus in advanced renal-cell carcinoma. N Engl J Med 2015;373:1803-13.

2 Powles T, Staehler M, Ljungberg B, et al. Updated EAU guidelines for clear cell renal cancer patients who fail VEGF targeted therapy. Eur Urol 2016;69:4-6.

3 NCCN. Clinical practice guidelines in oncology kidney cancer version 22017.

4 Hsieh JJ, Purdue MP, Signoretti S, et al. Renal cell carcinoma. Nat Rev Dis Primers 2017;3:1-19. 
5 Qing Y, Li Q, Ren T, et al. Upregulation of PD-L1 and APE1 is associated with tumorigenesis and poor prognosis of gastric cancer. Drug Des Devel Ther 2015;9:901-9.

$6 \mathrm{Mu} \mathrm{C}-\mathrm{Y}$, Huang J-A, Chen Y, et al. High expression of PD-L1 in lung cancer may contribute to poor prognosis and tumor cells immune escape through suppressing tumor infiltrating dendritic cells maturation. Med Oncol 2011;28:682-8.

7 Hirai M, Kitahara H, Kobayashi Y, et al. Regulation of PD-L1 expression in a high-grade invasive human oral squamous cell carcinoma microenvironment. Int J Oncol 2017;50:41-8.

8 Chen Y, Wang Q, Shi B, et al. Development of a sandwich ELISA for evaluating soluble PD-L1 (CD274) in human sera of different ages as well as supernatants of PD-L1+ cell lines. Cytokine 2011:56:231-8.

9 lacovelli R, Nolè F, Verri E, et al. Prognostic role of PD-L1 expression in renal cell carcinoma. A systematic review and meta-analysis. Target Oncol 2016;11:143-8.

10 Eisenhauer EA, Therasse P, Bogaerts J, et al. New response evaluation criteria in solid tumours: revised RECIST guideline (version 1.1). Eur J Cancer 2009;45:228-47.

11 Frigola X, Inman BA, Lohse CM, et al. Identification of a soluble form of $\mathrm{B} 7-\mathrm{H} 1$ that retains immunosuppressive activity and is associated with aggressive renal cell carcinoma. Clin Cancer Res 2011;17:1915-23. 